\title{
LA APROPIACIÓN DE LA CÁTEDRA DE LA PAZ A PARTIR DE LAS VOCES DE LOS ESTUDIANTES
}

Ricardo Ramírez Valencia*, David Gutiérrez Hernández**, Laura Vélez Rivera***

\section{Resumen}

El presente proyecto parte de los enfoques socioculturales, y la mirada de las situaciones educativas como eje integral y no como componentes apartes. Se analizan también los cambios que se generan a partir de la incorporación de las voces de los estudiantes mediante la posibilidad de la participación y elección que puedan tener ellos en sus procesos educativos (Rudduck y Flutter, 2007), además de cómo esto posibilita también la apropiación de los saberes (Rogoff, 1997), en específico de la asignatura Cátedra de la Paz. Este proyecto se pretende realizar desde la investigación-acción-educativa (IAE), en donde se trabaja directamente con las necesidades de la comunidad.

Palabras clave: apropiación, Cátedra de la Paz, participación, situación educativa, voces de los estudiantes.

* Estudiante de Psicología, VI semestre, Fundación Universitaria del Área Andina. Correo: rramirez43@estudiantes.areandina.edu.co

* Investigador principal. Docente del programa de Psicología, Grupo de investigación PAIDEIA, Fundación Universitaria del Área Andina. Correo: dgutierrez84@areandina. edu.co

*** Coinvestigadora, Corporación Universitaria Minuto de Dios. Correo: Ivelezriver@ uniminuto.edu.co 


\section{Introducción}

Primeramente, y en orden de entender el rol que define a los individuos dentro de las situaciones educativas, es relevante advertir que antes del Renacimiento la infancia se configuraba dentro de dinámicas similares a las de los adultos; los niños no se diferenciaban de los adultos, ya que compartían con ellos todo tipo de prácticas. Así, durante este movimiento específico comenzó a constituirse una infancia segregada de la vida de los adultos (Ariés, 1972, citado en Perinat, 2007), tomando la infancia desde el abordaje de Baquero (2006), en el que se determina no como un infante adulto, capaz de realizar las diferentes actividades de la cotidianidad, sino como un individuo dependiente, el cual tiene necesidades importantes y debe ser tomado como prioridad en su entorno general, desenvolviéndose esencialmente en su hogar, pero al mismo tiempo en la escuela a la que asiste, en donde se determina como una artificio social basado en las pautas de crianza y con la finalidad de crear un sistema disciplinario para los infantes.

Estas características terminarían por conformar, además, el papel del alumno en la escuela como infante moderno, un ser en la que la heteronomía se estructura como la característica central y cuyo principal deber se remite a la asimilación de conocimientos (Baquero y Narodowski, 1994). Es precisamente a esto, a lo que se refiere la metáfora de "la adquisición" (Baquero, 2006; Rogoff, 1997), y desde la que se enmarca el propósito primordial de la escuela.

Complementario a estas propiedades de los individuos en posición de alum- nos, el dispositivo escolar en parte afirmado por la psicología, idea una concepción de desarrollo en la que existe un inicio y un final, una especie de vía en la que se avanza de forma progresiva, y lo que es aún más paradójico, una línea de desarrollo sobre la cual los sujetos marchan de forma simultánea y homogénea (Baquero, 2006). Sobre las consideraciones anteriores, los desvíos en los cursos de desarrollo preestablecidos se encuadran bajo la etiqueta de fracaso escolar.

Junto a esto, Riviere (1983) expone la relación entre las peculiares dinámicas y procesos del sistema educativo, y el fracaso escolar como fenómeno. Producto de este análisis son sus diez mandamientos cognitivos de la escuela, resumidos básicamente en el primero y el último: "Desvincularás gran parte de tu pensamiento de los propósitos e intenciones humanas [...] y, para colmo, deberás parecer un niño interesado y competente" (Riviere, 1983, p. 1). De manera especial, esta desvinculación a la que hace referencia el autor, se refiere al carácter descontextualizado de los aprendizajes en las situaciones educativas, a ilustrar, la enseñanza de la Cátedra de la Paz, supone un aprendizaje de sucesos y aspectos pasados que en muy pocas ocasiones es explicado con relación a sus repercusiones en el futuro y más específicamente, en aspectos como la resolución de problemas.

A lo largo de los planteamientos realizados, ha sido posible reconocer la naturaleza y dinámicas de los complejos procesos alrededor de las prácticas educativas, así como su papel en la conformación del rol que hoy en 
día desenvuelven los estudiantes. Así pues, se plantea la ineludible labor de buscar estrategias que potencien más allá de los aprendizajes, una relación de los mismos con el desarrollo multidireccional y variado de los sujetos, al tiempo que promueve roles más activos y mayores formas de participación (Baquero, 2006).

Entender las posibilidades de los individuos como sujetos activos y partícipes de su propio aprendizaje (Erausquin, 2010; Zmuda, 2008), al ritmo de una reorganización en las formas de participación por medio de la incorporación de las voces de los estudiantes (Mateos, 2008; Rudduck y Flutter, 2007; Susinos y Ceballos, 2012), más que en aras de consultoría (Lanskey y Rudduck, 2010), en posibilidades de acción, constituye el propósito de la presente investigación. Tal y como propone Sandoval (2011): "Escuchar las voces de nuestros alumnos no es simplemente ofrecer la oportunidad para que éstos puedan comunicar sus ideas y opiniones, sino ser conscientes del papel que pueden tener a la hora de contribuir a los cambios educativos" (p. 116).

Finalmente, y siguiendo a Rogoff, una mayor implicación de los estudiantes en las prácticas tanto siendo parte como tomando parte, se traduce en apropiación participativa que transforma los individuos y prepara sus participaciones futuras en actividades relacionadas (Rogoff, 1997).

A su vez, también se elige la Cátedra de la Paz a partir del Decreto 1038 de 2015, "por el cual se reglamenta la Cátedra de la Paz", en el que todas las instituciones públicas y privadas del país deben implementar la Cátedra de la Paz en sus currículos $\mathrm{y}$ estos deben apuntar a fomentar la apropiación de conocimiento y competencias sobre el territorio, la cultura, la memoria histórica, entre otros.

Se tiene en cuenta que la metodología por la cual los estudiantes aprenden las temáticas propuestas en la asignatura de la Cátedra de la Paz, es a través del sistema tradicionalista del dispositivo escolar, un artificio creado en la modernidad con la finalidad de un sistema disciplinar al infante (Baquero, 2006). De manera histórica, la posición que ha tenido el alumno en los contextos educativos ha sido remitida a una posición carente, que precisa de protección y cuidados, en semejanza a la posición de infante moderno. De este modo, la escuela ha proporcionado todos los recursos para la construcción de una población escolar deseada, y que consecuentemente ha generado en los alumnos una posición pasiva a la espera de contenidos proporcionados por un dispositivo escolar (Baquero y Narodowski, 1994) en el que prima el carácter simultáneo y gradual (Baquero, 2006) tanto de los saberes como del desarrollo de los individuos que forman parte de las prácticas.

Por lo explícito anteriormente, es fundamental la implementación de la apropiación a los contenidos referidos a la Cátedra de la Paz en las 
instituciones educativas, debido que la práctica educativa estaría entendida desde la heterogeneidad del estudiante y los distintos planos en donde se resalta el rol activo del estudiante; reiterando la importancia de las relaciones interpersonales y cambios en un sentido interpersonal para tareas ulteriores de temática similar. Una vez conseguido esto, los propósitos como las soluciones y herramientas para el alumnado, familiarizarse con el contexto social y situaciones actuales, asimismo al apropiarse de sus derechos y temas relacionados con el tejido histórico de Colombia, desarrollándose en el estudiante conocimientos bases para una buena participación en la sociedad.

Es, precisamente, la idea del desarrollo sobre expectativas de ritmos iguales, la que se pretende superar - no en términos de competencia, sino en pro de avanzar - a través de los enfoques socioculturales. En primer lugar, con su rasgo distintivo del énfasis en el origen social de los fenómenos psicológicos (Vygotsky, 1978), y, en segundo lugar, al postular la premura de un cambio en las unidades de análisis que permita entender todo el entramado de las situaciones (Baquero, 2009).

Se tiene, entonces, que el propósito de la presente investigación es analizar los aportes que se puedan generar a partir de la incorporación de las voces de los estudiantes, para contribuir a la apropiación de la Cátedra da. Para esto se tiene también tres objetivos específicos, comenzando con la descripción de las situaciones educativas que ya estén presentes en la asignatura de Cátedra de la Paz, luego incorporar las voces de los estudiantes con procesos de elección y participación $y$, por último, identificar qué aportes se presentan en las situaciones educativas al incorporar dichas voces.

De ese modo, se entiende que el desarrollo cognitivo, como apropiación participativa, requiere prestar atención a las relaciones sociales que se dan en las actividades socioculturales como las prácticas educativas. En el plano personal, la apropiación supone un proceso de conversión que parte de la implicación y participación de los individuos en las prácticas, y que se traduce en transformación y preparación orientadas al futuro (Rogoff, 1997); partiendo así de la teoría sociocultural desde la que el desarrollo de los individuos es inseparable de los procesos sociales, así en la explicación del mismo se debe pasar del sujeto a las prácticas en las que está implicado. Además, postula la construcción de procesos psicológicos de tipo superior-avanzados a partir de especificidades de la cultura, como lo son los procesos de escolarización (Vygotsky, 1978). Las voces de los estudiantes, comprendida desde Rudduck y Flutter (2007), plantean que las iniciativas en el movimiento de promover la voz de los estudiantes, han apuntado al liderazgo y a principios democráticos. Existe una tercera interpretación que 
aún es embrionaria, y es la que apunta al aprendizaje desde la experiencia guiada a sus miembros.

\section{Metodología, enfoque y tipo de investigación}

La presente investigación es de tipo cualitativo con enfoque de la investigación-acción-educativa desde lo que propone Elliot (2005), y en la que el rol del docente es también como investigador, trabajando de la mano con las necesidades de la comunidad que serían, en este caso, tres instituciones educativas del departamento de Risaralda y en donde los estudiantes pasan a ser agentes activos donde la direccionalidad de la enseñanza-aprendizaje cambiará atendiendo al proceso formativo compartido con los alumnos, quienes podrán seguir su propio camino en el desarrollo de su formación (Ventosa, 2016). Siguiendo el proceso de investigación-acción, según Kemmis y McTaggart (1992), consta de cuatro fases: diagnóstico; planificación de las acciones; actuación y observación y, por último, reflexión o evaluación. En el desarrollo de estas fases se utilizarán las siguientes técnicas de recolección de datos: grupos de discusión, entrevistas, observación participante, análisis de documentos institucionales $y$, como apoyo, se usarán los diarios de campo y fotografías.

\section{Discusión}

En línea con los objetivos específicos, se tiene la descripción de las situaciones educativas con base en las formas de participación, las reglas, los roles y el uso de los instrumentos; además de la generación de estrategias para la incorporación de las voces de los estudiantes dentro del desarrollo de las cosas y en línea con las planificaciones del docente; posterior a esto, la identificación de los cambios generados a partir de la incorporación de las voces de los estudiantes.

Como indica Prieto (2005), la calidad educativa ha estado restringida a lograr que los alumnos aprendan lo establecido en los planes y programas curriculares. Se ha olvidado que la calidad educativa está indisolublemente vinculada a la calidad de la formación de los estudiantes, personas autónomas responsables de sus propios procesos formativos. Lo anterior demanda urgentes transformaciones en las que se deban implementar procesos escolares que promuevan su desarrollo personal y la consiguiente emancipación, lo que implica promover su activa participación en la Cátedra de la Paz. Esto ayuda a mejorar los procesos formativos y promover cambios. Es necesario escuchar las voces de los estudiantes acerca de su calidad, concederles la posibilidad de realizar propuestas y tomar decisiones al respecto. Ellos tienen mucho que decir sobre el tema $y$, dado que son los que producen los resultados escolares, resulta fundamental su involucramiento para mejorarlos.

\section{Referencias}

1. Baquero, R., \& Narodowski, M. (1994). ¿Existe la infancia? Revista del Instituto de Investigaciones en Ciencias de la Educación de la Universidad de Buenos Aires, 3(6), 61-67.

2. Baquero, R., \& Terigi, F. (1996). En búsqueda de una unidad de análisis del aprendizaje escolar. Dossier "Apuntes 
pedagógicos" de la revista Apuntes. UTE/ CTERA. Buenos Aires.

3. Baquero, R. (2006). Sujetos y aprendizaje. Ministerio de Educación. Argentina.

4. Baquero, R. (2009). Desarrollo psicológico y escolarización en los enfoques socioculturales: nuevos sentidos de un viejo problema. Avances en Psicología Latinoamericana. Bogotá, 27(2), 263-280.

5. Elliot, J. (2005). El cambio educativo desde la investigación-acción. Madrid.

6. Erausquin, C. (2010). Adolescencias y escuelas: interpelando a Vygotski en el siglo XXI. Revista de Psicología, segunda época, 11.

7. Kemmis, S., \& McTaggart, R. (1992). Cómo planificar la investigación-acción. https://es.scribd.com/document/344401915/ Kemmis-E-y-McTaggart-1992-Como-Planificar-Investigacion-Accion-Cap-1-Apendices-b-y-c

8. Lanskey, C., \& Rudduck, J. (2010). Leadership and student voice. https://doi. org/10.1016/B978-0-08-044894-7.00458-9

9. Mateos Blanco, T. (2008). La percepción del contexto escolar. Una imagen construida a partir de las experiencias de los alumnos. Cuestiones Pedagógicas, 19, 285-300.

10. Parody, G. (2010). El Decreto 1038 de 2015: en búsqueda de la paz. https://repository.usergioarboleda.edu.co/ bitstream/handle/11232/1047/Seguridad\%20y\%20Democracia\%2010\%203. pdf? sequence=1\&isAllowed=y

11. Perinat, A. (2007). Psicología del desarrollo. Un enfoque sistémico (vol. 83). Barcelona.
12. Prieto, M. (2005). La participación de los estudiantes: ¿un camino hacia su emancipación? Theoria, 14(1), 27-36.

13. Riviere, A. (1983). ¿Por qué fracasan tan poco los niños? Cuadernos de Pedagogía, 104, 7-12.

14. Rogoff, B. (1997). Los tres planos de la actividad sociocultural: "apropiación participativa", "participación guiada" y "aprendizaje". En: Wertsch, J., del Río, P. y Álvarez, A. (eds.). La mente sociocultural. Aproximaciones teóricas y aplicadas, Madrid.

15. Rudduck, J., \& Flutter, J. (2007). Cómo mejorar tu centro escolar dando la voz al alumnado. Madrid.

16. Sandoval, M. (2011). Aprendiendo de las voces de los alumnos y alumnas para construir una escuela inclusiva. REICE. Revista Iberoamericana sobre Calidad, Eficacia y Cambio en Educación, 9(4), 114-125.

17. Susinos Rada, T., \& Ceballos López, N. (2012). Voz del alumnado y presencia participativa en la vida escolar. Apuntes para una cartografía de la voz del alumnado en la mejora educativa. https://www.educacionyfp.gob.es/dam/jcr:45ae6a89-9fdb4cd9-bee4-3e9e1c93b1cd/re35902.pdf

18. Ventosa, V. (2016). Manual de técnicas de participación y desarrollo grupal. Madrid.

19. Vygotsky, L. (1978). El desarrollo de los procesos psicológicos superiores. Barcelona.

20. Zmuda, A. (2008). Springing into. Educational Leadership, 66(3), 38-42. 